Article

\title{
Transcatheter Tricuspid Valve-in-Valve Replacement by Transatrial Approach
}

\author{
Grigore Tinica ${ }^{1,2}$, Igor Nedelciuc ${ }^{1}$, Iulian Rotaru ${ }^{1,2}$, Flavia Catalina Corciova ${ }^{1}$, \\ Dumitru Grajdianu ${ }^{1}$, Andrei Tarus ${ }^{1,2}$, Mihail Enache ${ }^{1,2}$, Raluca Ozana Chistol 1,2,*, \\ Maria Luiza Cobzeanu ${ }^{2,3, *}$ and Cristina Furnica ${ }^{2,4}$ \\ 1 Cardiovascular Diseases Institute, 700503 Iasi, Romania; grigore.tinica@umfiasi.ro (G.T.); \\ igor.nedelciuc@cardioiasi.ro (I.N.); iulian-g-rotaru@d.umfiasi.ro (I.R.); flavia.corciova@cardioiasi.ro (F.C.C.); \\ dumitru.grajdianu@cardioiasi.ro (D.G.); andrei-v-tarus@d.umfiasi.ro (A.T.); mihail.enache@umfiasi.ro (M.E.) \\ 2 Faculty of Medicine, Grigore T. Popa University of Medicine and Pharmacy, 700115 Iasi, Romania; \\ cristina.furnica@umfiasi.ro \\ 3 Department of Radiology, Sf Spiridon University Hospital, 700111 Iasi, Romania \\ 4 Institute of Forensic Medicine, 700455 Iasi, Romania \\ * Correspondence: raluca-ozana.chistol@umfiasi.ro (R.O.C.); maria-luiza.g.baean@d.umfiasi.ro (M.L.C.)
}

Received: 1 August 2020; Accepted: 17 September 2020; Published: 18 September 2020

Featured Application: Novel application of valve-in-valve therapy of degenerated bioprosthetic cardiac valves.

\begin{abstract}
Reoperation on the tricuspid valve after prior heart valve surgery is associated with an increased operative risk due to a poor clinical status with severe heart failure and late presentation. Transcatheter tricuspid valve-in-valve implantation emerged as an attractive alternative to a high-risk redo surgery. The authors report a case of successful treatment of a failed bioprosthetic tricuspid valve in a 58-year-old woman with severe heart failure, decompensated cardiac cirrhosis and atrial fibrillation using transcatheter tricuspid valve-in-valve implantation of a Sapien 3 valve (Edwards Lifesciences, Irvine, California) via a transatrial approach. This case demonstrates the efficiency of this novel approach for the treatment of dysfunctional surgical tricuspid bioprosthetic valves and the technical feasibility and safety of a rarely used route.
\end{abstract}

Keywords: tricuspid valve-in-valve; transapical; heart failure; heart valve surgery; endovascular; interventional

\section{Introduction}

Tricuspid regurgitation (TR) is a common valvular heart disease affecting over 70 million people worldwide. According to the 2017 ESC/EACTS guidelines for the management of valvular heart disease, reoperation on the tricuspid valve (TV) after prior heart valve surgery carries an increased operative risk due to a poor clinical status with severe heart failure and late presentation [1].

As TR is associated with an unfavorable outcome in this group of patients and valve-in-valve transcatheter aortic valve implantation (ViV-TAVI) proved its safety and efficiency in treating failed surgical aortic bioprostheses [2-4], transcatheter tricuspid valve-in-valve (TTVIV) implantation emerged as an attractive alternative to high-risk redo surgery.

Roberts et al., 2010, reported the first TTVIV case treated with a Melody valve (Medtronic, Minneapolis, MN, USA) implanted via a transjugular approach [5]. McElhinney et al., 2019, published the mid-term results of a multicenter registry study on 306 patients who underwent TTVIV using Melody (Medtronic, Dublin, Ireland) or Sapien 3/XT (Edwards Lifesciences, Irvine, CA, USA) valves [6]. 
In the current paper we present the case of a patient who benefited from TTVIV via a rarely used access route as a salvage intervention for treating the failure of a degenerated tricuspid bioprosthesis with severe tricuspid stenosis and regurgitation. The patient gave her consent for publishing the case.

\section{Case Presentation}

A 58-year-old woman was referred to the Cardiac Surgery Department (Cardiovascular Diseases Institute, Iasi, Romania) for fatigue, severe dyspnea with orthopnea and progressive refractory anasarca (10 cm bilateral pleural effusion at admission). The patient was previously diagnosed with New York Heart Association (NYHA) IV heart failure, decompensated cardiac cirrhosis, splenomegaly, umbilical hernia, permanent atrial fibrillation and moderate to severe pulmonary hypertension associated to the dysfunction of a tricuspid bioprosthesis.

At age 39 she underwent surgical aortic valve replacement with a $23 \mathrm{~mm}$ Edwards MIRA Mechanical Bileaflet Valve (Edwards Lifesciences, Irvine, CA, USA), mitral valve replacement with a $29 \mathrm{~mm}$ Sorin Bicarbon Mechanical Bileaflet Valve (Sorin Biomedica Cardio, Saluggia, Italy) and tricuspid valve replacement with a $33 \mathrm{~mm}$ Sorin Pericarbon Bioprosthesis (Sorin Biomedica Cardio, Saluggia, Italy) for rheumatic heart valve disease. Because of atrial fibrillation and triple valve replacement, the patient was on oral anticoagulation with acenocoumarol (vitamin-K antagonist).

The transoesophageal echocardiography (TEE) performed upon admission revealed normofunctional aortic and mitral valves, severe bi-atrial dilatation, a calcified tricuspid bioprosthesis with severe stenosis and regurgitation leading to a mild right ventricular dysfunction.

The mean tricuspid transvalvular gradient was $13 \mathrm{mmHg}$; the peak transvalvular gradient $17 \mathrm{mmHg}$; peak velocity $2 \mathrm{~m} / \mathrm{s}$; PHT (pressure half time) $626 \mathrm{~ms}$; and the tricuspid orifice area $0.4 \mathrm{~cm}^{2}$ (Figure 1). The right atrium was severely dilated with spontaneous contrast inside; bilateral pleural effusion and ascites were also present.

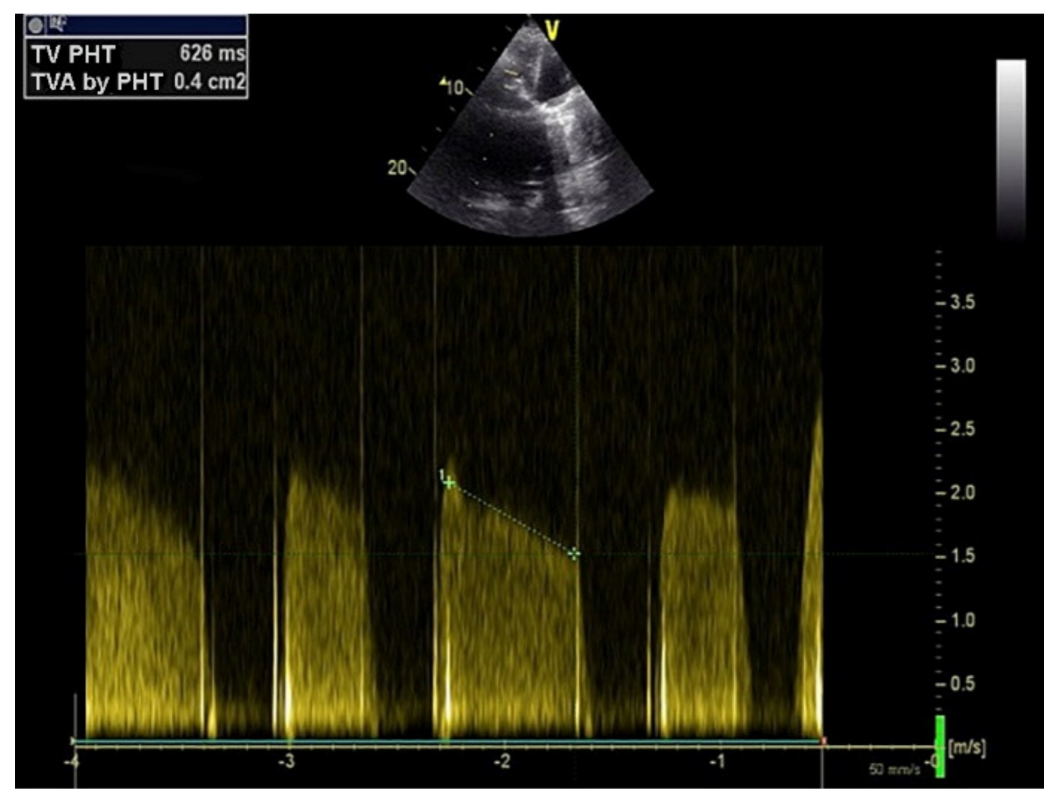

Figure 1. Transoesophageal echocardiography mid-oesophageal four-chamber incidence showing severe tricuspid stenosis with pressure half time (PHT) $626 \mathrm{~ms}$ and tricuspid valve area (TVA) $0.4 \mathrm{~cm}^{2}$.

The patient presented multiple comorbidities and the Heart Team considered her at prohibitive surgical risk according to the Society of Thoracic Surgeons (STS) score (17.92\% for mortality and $48 \%$ for morbidity), European System for Cardiac Operative Risk Evaluation (Euroscore) II (22.2\%) and Charlson comorbidity index score [7].

In this context, the Heart Team opted for a transatrial TTVIV replacement with a $29 \mathrm{~mm}$ Edwards Lifesciences Sapien 3 valve (Edwards Lifesciences, Irvine, CA, USA). 
TTVIV sizing was based on the cardiac computed tomography angiography (CCTA) evaluation of the pre-existing bioprosthesis and not on the reported size of the Sorin Pericarbon bioprosthesis (Sorin Biomedica Cardio, Saluggia, Italy) because, according to Praz et al. [7], the true internal diameter of the pre-existing bioprosthesis (of interest for TTVIV) is generally 1 to $2 \mathrm{~mm}$ smaller than the one reported by the manufacturer (Figure 2).

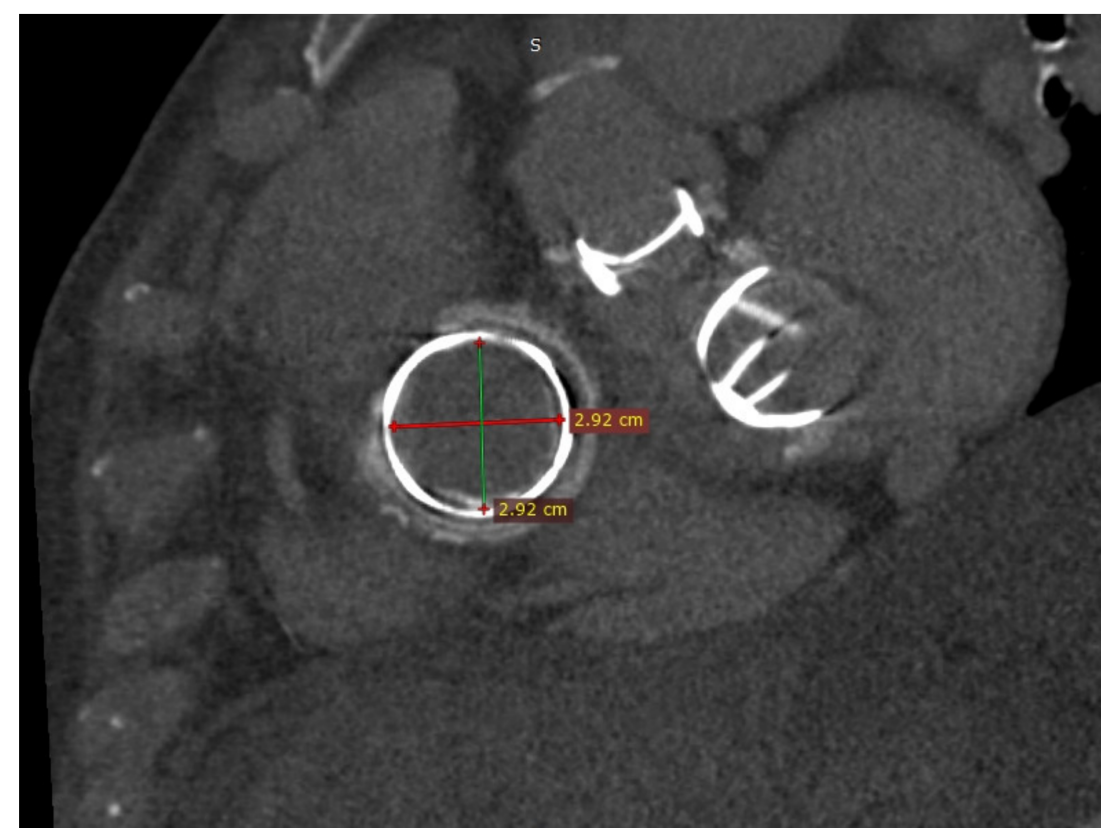

Figure 2. Cardiac computed tomography angiography measurements of the pre-existing tricuspid bioprosthesis.

In our case, the $33 \mathrm{~mm}$ Sorin Pericarbon bioprosthesis (Sorin Biomedica Cardio, Saluggia, Italy) proved to have a $29 \mathrm{~mm}$ internal diameter.

After informed consent was obtained, the patient received general anesthesia with endotracheal intubation, and the approach to the tricuspid valve was performed by right lateral thoracotomy in the fourth intercostal space. The cardiac surgery team and an extracorporeal membrane oxygenation were on standby in the event of an acute complication that would impose open heart surgery.

The right lung was retracted to expose the pericardium and 2 Prolene 3.0 (Ethicon, Somerville, NJ, USA) purse-string sutures reinforced with pledges were placed in the lateral wall of the right atrium. A bolus of $5000 \mathrm{IU}$ of heparin was administered intravenously prior to puncturing the right atrium in the middle of the purse-string sutures. The interventional cardiologist was guided by TEE during the whole procedure. The dysfunctional tricuspid valve was accessed with a soft J-guidewire inserted through the puncture and advanced to the right ventricle. Afterwards, a 7F sheath with a pigtail catheter was mounted with subsequent removal of the guidewire. A Confida (Medtronic, Minneapolis, MN, USA) stiff wire with a preshaped loop was inserted through the pigtail to the apex of the right ventricle (Figure 3) to ensure stability for transcatheter valve crossing from the right atrium to the right ventricle. 


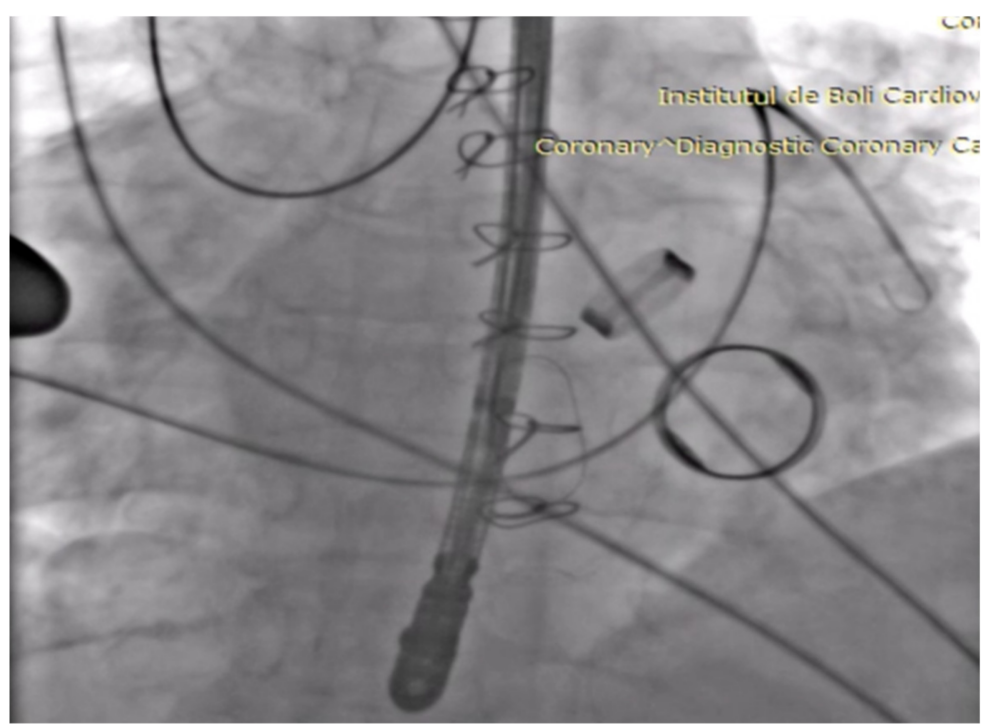

Figure 3. Fluoroscopic image showing the mechanical mitral and aortic prostheses, the tricuspid bioprosthesis, prior pacing leads and the stiff wire.

The 7F sheath and the pigtail were removed, and a 16F Edwards eSheath Introducer was inserted. The Edwards Delivery System with the Sapien 329 mm valve (Edwards Lifesciences, Irvine, CA, USA) was passed through the introducer. Under fluoroscopic (C-arm perpendicular to the bioprosthetic valve annulus) and TEE guidance, the system was advanced to the annulus of the degenerated bioprosthetic valve that served for accurate alignment and positioning of the transcatheter valve. Fine adjustment was carried with the balloon catheter (pulling and rotating to optimal centering of the valve). Prior to valve deployment, rapid pacing was initiated using the Confida guide wire as a cathode while the anode was placed in the subcutaneous tissue of the right inguinal region. The Sapien 3 valve was then deployed by expanding the balloon to the nominal pressure value with excellent results (Figure 4).

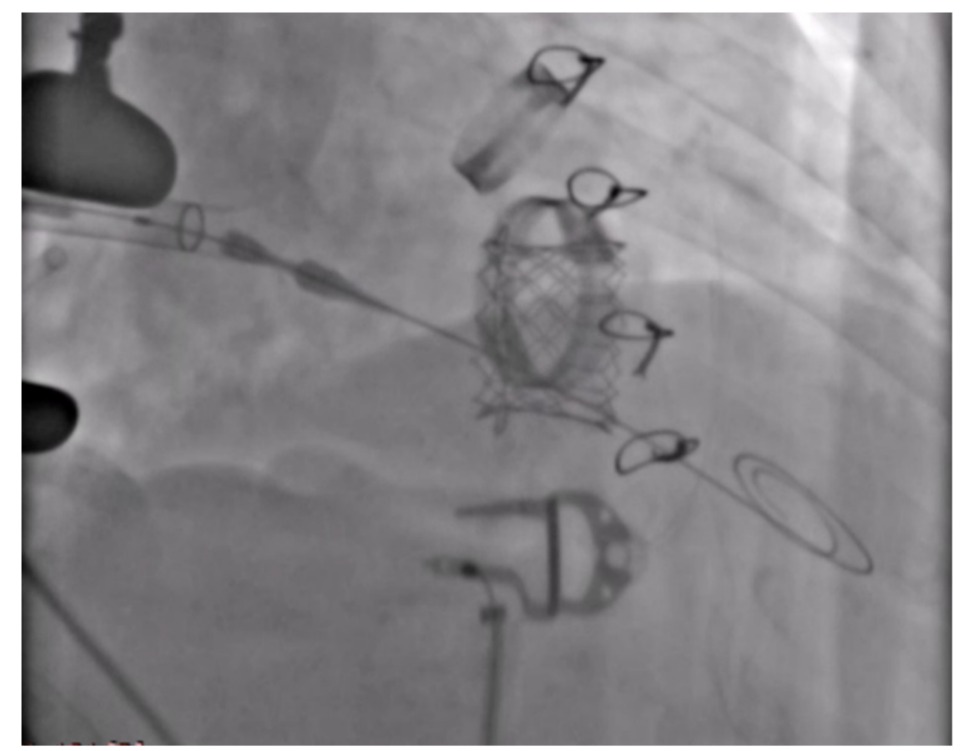

Figure 4. Fluoroscopic image showing the normofunctional Edwards Sapien 3 valve-in-valve after deployment.

The delivery system, the introducer sheath and the guide wire were removed after confirming the position and the competence of the TTVIV by TEE (Figure 5). After extracting all materials, the two purse strings were ligatured followed by meticulous hemostasis. The right lung was reinflated using 
the Valsalva maneuver and a drainage catheter was inserted in the right costodiaphragmatic recess before closing the thoracotomy site. Postoperative evolution was uneventful, and the patient was discharged after two weeks with NYHA class II heart failure compared to IV at admission.

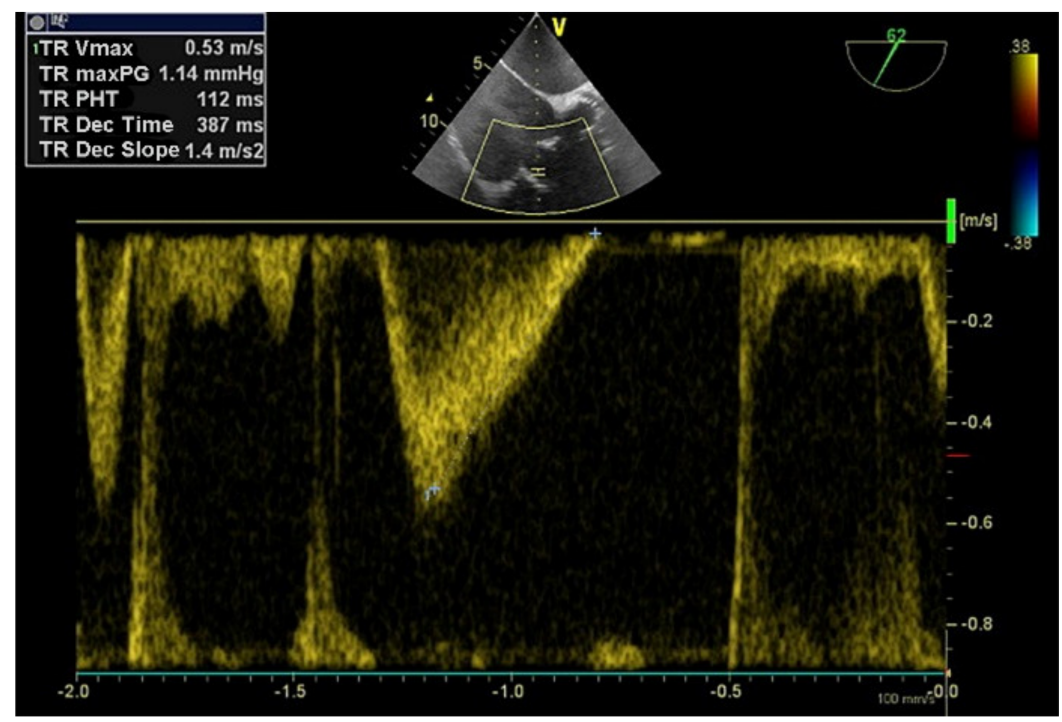

Figure 5. Transoesophageal echocardiography mid-oesophageal four-chamber incidence confirming the normal functioning the tricuspid bioprosthetic valve-in-valve with a maximum transvalvular gradient of $1.14 \mathrm{mmHg}$ and a peak velocity of $0.53 \mathrm{~m} / \mathrm{s}$, suggesting substantial reduction in gradient severity after transcatheter valve implantation.

The patient was re-evaluated at one month and three months after the intervention. TTE revealed normofunctional aortic, mitral and tricuspid valves, bi-atrial dilatation, and a residual systolic dysfunction of the right ventricle. The patient maintained NYHA class II heart failure, permanent atrial fibrillation, and cardiac cirrhosis. Bilateral pleural effusion persisted at one month $(8 \mathrm{~cm}$ on the right and $9 \mathrm{~cm}$ on the left) and markedly regressed at three months $(2 \mathrm{~cm}$ on the right and absent on the left) after the intervention. Ascitic fluid persisted at one month and was absent at three months.

\section{Discussion}

Transatrial approach for TTVIV is used in less than $4 \%$ of cases [6-8] as it is considered more invasive and technically difficult compared to the transfemoral and transapical route.

Praz et al., Sanon et al. and Mick et al. consider that the transjugular access offers the most direct approach, especially in horizontally oriented TVs followed by the transfemoral route also feasible in two thirds of patients mainly with more vertically oriented TVs because of the technical advances in catheter steerability [9-11]. These two routes are preferred by most teams $(96.72 \%$ of patients analysed by McElhinney et al. in 2016) as they are the least invasive and do not need a surgical intervention for access [7].

The current case proves the safety, feasibility, and efficiency of the rarely used transatrial approach and pleads for its use in patients with unfavorable venous anatomy and severe stenosis of the degenerated tricuspid bioprosthesis complicating the access with the delivery system. The transjugular route did not offer a good angle to approach the TV in our patient and the marked angulation of the inferior vena cava when entering the right atrium precluded the transfemoral access to the degenerated TV bioprosthesis.

For this procedure, we chose the Edwards Sapien 3 bioprosthesis, approved for valve-in-valve procedures, given the prior experience with this type of valve implanted in aortic position (transcatheter aortic valve implantation-TAVI) in more than 100 cases and its particular design associated with a low risk of paravalvular regurgitation after deployment. 
Tricuspid valve-in-valve deployment is particularly difficult as transvenous pacemakers generally implanted across the valve could be dislodged when manipulating the delivery system. For this reason, we opted for a different method of rapid ventricular pacing using an intraventricular stiff guidewire as cathode with the anode placed subcutaneously. Rapid ventricular pacing was necessary to allow an accurate deployment in the setting of elevated right atrial pressures. We consider that transatrial access and direct pacing on Confida guidewire could increase procedural success. In the absence of ventricular pacing, TTVIV deployment is difficult.

Particular attention was paid to manipulating catheters, the delivery system, and the guide wire, as the Sapien 3 valve was originally designed to navigate the aorta and to be deployed in the aortic annulus. The Confida stiff wire ensured the necessary support while navigating through the right atrium and the tricuspid valve, the steering potential of the system reducing trauma to right heart chambers.

McElhinney et al. performed the single multi-center study on the short-term (2016) and mid-term (2019) outcomes of TTVIV [6,7]. They analyzed 306 patients who benefited both from Medtronic and Edwards valves implanted by tranjugular, transfemoral or, exceptionally, by transatrial approach. The patients were followed for a median of 15.9 months after implantation ( 0.1 to 90 months) in 80 centers. They proved the favorable outcome of the procedure as the 3-year mortality, reintervention, and TTVIV-related adverse outcomes rates were $17 \%, 12 \%$, and $8 \%$ considering the severe status of patients that need TTVIV (NYHA III-IV heart failure, prior heart surgery, cardiovascular comorbidities, decompensated status).

Given the severity of the disease and the poor outcome in the absence of redo valve replacement, the results are encouraging. TTVIV related complications (endocarditis, valve thrombosis) were reported in 16 cases $(5.23 \%)$, valve function remaining stable in most patients [6,7]. The durability of the TTVIV is yet to be established as no long-term follow-up studies were published.

The procedure could be optimized by designing specific valves and delivery systems as the currently used ones are not imaged specifically for this purpose. Adequate medical imaging and anatomic planning are the cornerstone for procedural success as transcatheter valve replacement emerges as the salvage choice in extreme situations. With technological progress, newer generation devices and increased experience, TTVIV outcomes are expected to further improve in the years to come.

\section{Conclusions}

Transatrial TTVIV is technically feasible and safe in patients with severe stenosis of the degenerated tricuspid bioprosthetic valve and unfavorable anatomy precluding transfemoral and tranjugular access. Direct pacing on the guidewire could increase procedural success by avoiding the insertion of additional catheters that could be dislodged when manipulating the delivery system.

Author Contributions: Conceptualization, G.T. and C.F.; methodology, G.T., A.T., M.E. and I.R.; investigation, A.T., I.R., F.C.C., I.N., D.G., M.E. and R.O.C.; resources, R.O.C. and M.L.C.; writing-original draft preparation, C.F., R.O.C., M.L.C.; writing-review and editing, G.T. All authors have read and agreed to the published version of the manuscript.

Funding: This research received no external funding.

Conflicts of Interest: The authors declare no conflict of interest.

\section{References}

1. Baumgartner, H.; Falk, V.; Bax, J.J.; De Bonis, M.; Hamm, C.; Holm, P.J.; Iung, B.; Lancellotti, P.; Lansac, E.; Rodriguez Munoz, D.; et al. 2017 ESC/EACTS Guidelines for the management of valvular heart disease. Eur. Heart J. 2017, 38, 2739-2791. [CrossRef] [PubMed]

2. Jones, J.M.; O'Kane, H.; Gladstone, D.J.; Sarsam, M.A.; Campalani, G.; MacGowan, S.W.; Cleland, J.; Cran, G.W. Repeat heart valve surgery: Risk factors for operative mortality. J. Thorac. Cardiovasc. Surg. 2001, 122, 913-918. [CrossRef] [PubMed] 
3. Wenaweser, P.; Buellesfeld, L.; Gerckens, U.; Grube, E. Percutaneous aortic valve replacement for severe aortic regurgitation in degenerated bioprosthesis: The first valve in valve procedure using the Corevalve Revalving system. Catheter Cardiovasc. Interv. 2007, 70, 760-764. [CrossRef] [PubMed]

4. Walther, T.; Kempfert, J.; Borger, M.A.; Fassl, J.; Falk, V.; Blumenstein, J.; Dehdashtian, M.; Schuler, G.; Mohr, F.W. Human minimally invasive off-pump valve-in-a-valve implantation. Ann. Thorac. Surg. 2008, 85, 1072-1073. [CrossRef] [PubMed]

5. Roberts, P.; Spina, R.; Vallely, M.; Wilson, M.; Bailey, B.; Celermajer, D.S. Percutaneous tricuspid valve replacement for a stenosed bioprosthesis. Circ. Cardiovasc. Interv. 2010, 3, e14-e15. [CrossRef] [PubMed]

6. McElhinney, D.B.; Aboulhosn, J.A.; Dvir, D.; Whisenant, B.; Zhang, Y.; Eicken, A.; Ribichini, F.; Tzifa, A.; Hainstock, M.R.; Martin, M.H.; et al. Mid-Term Valve-Related Outcomes After Transcatheter Tricuspid Valve-in-Valve or Valve-in-Ring Replacement. J. Am. Coll. Cardiol. 2019, 73, 148-157. [CrossRef] [PubMed]

7. McElhinney, D.B.; Cabalka, A.K.; Aboulhosn, J.A.; Eicken, A.; Boudjemline, Y.; Schubert, S.; Himbert, D.; Asnes, J.D.; Salizzoni, S.; Bocks, M.L.; et al. Transcatheter Tricuspid Valve-in-Valve Implantation for the Treatment of Dysfunctional Surgical Bioprosthetic Valves: An International, Multicenter Registry Study. Circulation 2016, 133, 1582-1593. [CrossRef] [PubMed]

8. Cheung, A.; Soon, J.L.; Webb, J.G.; Ye, J. Transatrial transcatheter tricuspid valve-in-valve technique. J. Card. Surg. 2012, 27, 196-198. [CrossRef] [PubMed]

9. Praz, F.; George, I.; Kodali, S.; Koulogiannis, K.P.; Gillam, L.D.; Bechis, M.Z.; Rubenson, D.; Li, W.; Duncan, A. Transcatheter Tricuspid Valve-in-Valve Intervention for Degenerative Bioprosthetic Tricuspid Valve Disease. J. Am. Soc. Echocardiogr. 2018, 31, 491-504. [CrossRef] [PubMed]

10. Sanon, S.; Cabalka, A.K.; Babaliaros, V.; Rihal, C.; Gafoor, S.; Webb, J.; Latib, A. Transcatheter Tricuspid Valve-in-Valve and Valve-in-Ring Implantation for Degenerated Surgical Prosthesis. JACC Cardiovasc. Interv. 2019, 12, 1403-1412. [CrossRef] [PubMed]

11. Mick, S.L.; Kapadia, S.; Tuzcu, M.; Svensson, L.G. Transcatheter valve-in-valve tricuspid valve replacement via internal jugular and femoral approaches. J. Thorac. Cardiovasc. Surg. 2014, 147, e64-e65. [CrossRef]

(C) 2020 by the authors. Licensee MDPI, Basel, Switzerland. This article is an open access article distributed under the terms and conditions of the Creative Commons Attribution (CC BY) license (http://creativecommons.org/licenses/by/4.0/). 